\title{
Tangible Computing in Everyday Life: Extending Current Frameworks for Tangible User Interfaces with Personal Objects
}

\author{
Elise van den Hoven and Berry Eggen \\ Eindhoven University of Technology, Department of Industrial Design, P.O. Box 513, \\ 5600MB Eindhoven, the Netherlands \\ \{e.v.d.hoven, j.h.eggen\}atue.nl
}

\begin{abstract}
This paper describes an extension to Ullmer and Ishii's TUIcategorization [41]. The reason for adding new categories is based on their omission of their associative TUIs and our work in this area of personal objects. The benefit of using personal objects instead of generic objects is that in the first instance users already have mental models or personal links between experiences, the related media and these objects. In addition, a Graspable or Tangible User Interface with personal objects can support existing media systems, instead of designing new ones that have to be learned by users.
\end{abstract}

\section{Introduction}

Over the last couple of years, it has been demonstrated that graspable, or tangible user interfaces (TUIs) make up a promising alternative for the 'traditional' omnipresent graphical user interface (GUI) (see [41] for an overview). TUIs integrate physical representation and mechanisms for interactive control into graspable user interface artefacts. This physical embodiment provides the user interface designer with new and powerful means to free user-system interaction from the confines of the desktop and merge it with the physical reality of everyday life.

The ambient intelligence paradigm of computing envisions a world in which electronically enriched environments have become sensitive and responsive to people and where electronic devices have disappeared into the background of everyday life. Such scenarios imply the need for new interaction styles that better match the skills humans use to interact with their natural environment.

Combining the need for new interaction styles that seamlessly merge into the physical world of everyday life and the promising possibilities TUIs offer in this respect, tangible approaches to user-system interaction seem an obvious choice to study in the context of ambient intelligence. Ullmer and Ishii [41] have proposed a conceptual framework that classifies many past and present systems according to a number of tangible interfaces characteristics. In recent years, this framework has become well-known and has served as a starting point for identifying and discussing the merits and demerits of TUIs. However, in our own research on tangible user interface for ambient intelligent environments ([18], [19], [20], [21]), we encountered difficulties 
when we tried to classify the 'tangibles' that were developed to support the interaction between people and their intelligent environments. Consider the following example:

You are on holiday and you visit this local market where you find a piece of art you like. You take it home and put it in your living room and each time you walk by you reminisce about that great time you had there. It is as if your memories come to life whenever you see this souvenir. And not only the souvenir creates this effect but also your grandfather's chair you inherited and the vase you got as a birthday present. It seems like objects make it possible to reexperience past events.

In this example, personal objects or souvenirs are automatically linked to memories or recollections and it is the owner's imagination that can relive the experience. Another option is to facilitate this experience by making a digital association with memory-cues, like for example digital photos. For a long time, people have been using self-made photos to re-experience their holidays, alone or with relatives and friends. Recently, digital cameras were introduced which are now rapidly replacing the analogue ones. Since a digital photo is virtual and difficult to grasp for some people, it might help if they could be associated with a physical object, such as the art-souvenir example mentioned above.

In our work on photo browsing in an ambient intelligent home environment, we explored the use of personal objects as the tangible part of the user interfaces for a Digital Photo Browser ([18], [19], [20], [21]). In trying to classify these personal objects using the framework of Ullmer and Ishii [41] we were not successful. It became apparent that the Ullmer and Ishii framework needed to be extended to accommodate the important class of personal objects in a satisfactory way.

In this paper we briefly discuss the Digital Photo Browser case study to illustrate the potential of using personal objects for interacting with ambient intelligent environments. Next, we review existing proposals for the classification of graspable user interfaces, including the Ullmer and Ishii framework. After proposing an extension to this framework, we conclude the paper with a general discussion and some conclusions.

\section{Case Study}

The context of this case study was focusing on autobiographical recollections, i.e. support in-home reminiscing. As these recollections are often associated with photos and artefacts, it was decided to build a Digital Photo Browser with graspable objects, in the shape of souvenirs, as physical carriers for virtual data. (The recently published Living Memory Box Project [36] is similar in this respect.) Another reason for using artefacts as Graspable User Interfaces was the result of a study [19] showing that souvenirs can serve as memory cues to its owners. Besides, the link between souvenirs in a Graspable UI and (digital) photos seems useful since often souvenirs are bought on a holiday, a moment in time and geographical location where the buyer of the souvenir also takes digital photos. This automatically establishes a mental link between the two types of media. At a later moment in time these digital photos can be easily linked to the personal souvenir. The user can do this with the Digital Photo Browser. 


\subsection{Digital Photo Browser}

In short, the Digital Photo Browser is a portable touch-screen device (both a Fujitsu Stylistic LT and a Philips DeXscape are used), which is connected via a $11 \mathrm{Mb} / \mathrm{s}$ wireless LAN link to a "fixed" PC, which runs various components, such as services, agents and general applications, which communicate using a service-discovery architecture (for detailed specifications, see [8]). In a real-life situation this fixed PC is in the attic or study but to the user all functionality appears to be in the portable device. In general this Digital Photo Browser can be used for storing, browsing and viewing digital photos. Adding new photos to the database is possible by means of an attached camera, by scanning or by uploading from other storage media.

Figure 1a shows a sketch of the Graphical UI of the Digital Photo Browser, which consists of three areas: 1 - an area on the left which shows a moving photo roll, 2 - a central area which allows enlarging individual photos, both landscape and portrait, 3 an area on the right where icons of the current user (3a), other display devices (3b) or detected graspable objects (3c) can be shown. In this context only area $3 \mathrm{c}$ is relevant, since it will show any graspable object detected by the system.
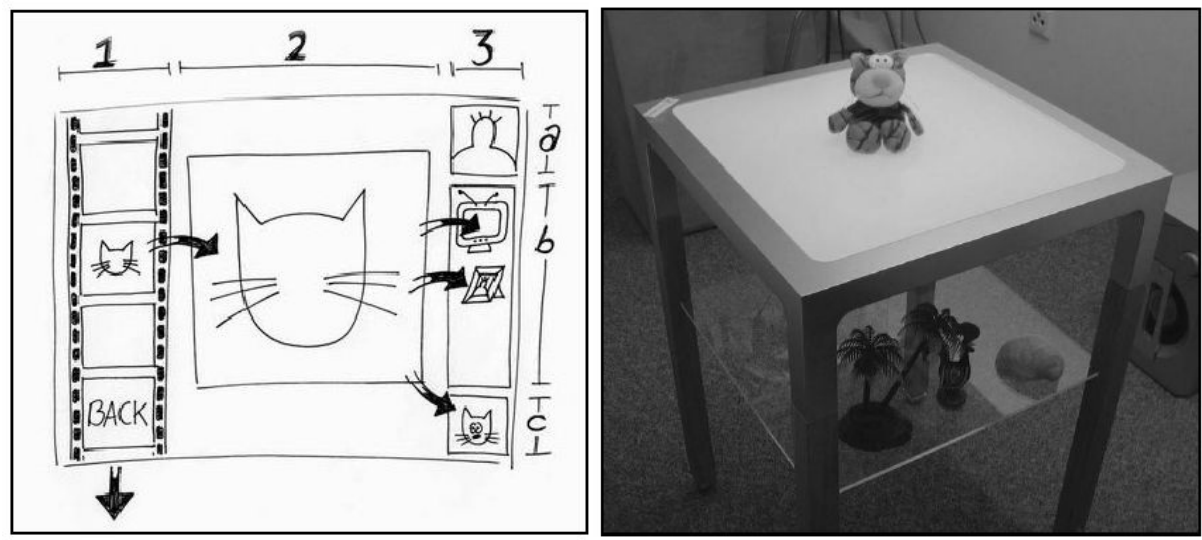

Fig. 1. (a) A sketch of the Photo-Browser user interface (for an explanation see text), and (b) the Chameleon Table and some tagged souvenirs.

\subsection{Personal Souvenirs}

How are these souvenirs used together with the Digital Photo Browser. First they are tagged with RFID-tags [33] and a special table (called the Chameleon Table) was constructed to detect those RFID-tags and to make clear to the user where and when tagged objects are recognized (see Fig. 1b). Once an RFID-tagged souvenir is recognized by the table, auditory feedback is provided and a thumbnail of the souvenir appears in area $3 \mathrm{c}$ on the GUI. Simultaneously, the content of area 1, which shows the moving photo roll, changes to the photos linked to the souvenir. New photos can be associated with the object by dragging thumbnails or enlarged photos to the icon of 
the object. Adding photos to the souvenir is done by dragging them from anywhere on the screen of the Digital Photo Browser to the icon of the souvenir, e.g., from photo albums displayed in the photo roll.

For this prototype the table only detects one object at the same time. This was decided not because of technical limitations but because otherwise it would require an object language, in order to explain which logical operators were used. This would make the object-device interaction less straightforward.

For more details on this case study, the working prototypes (see Fig. 2) or evaluations, see [19].

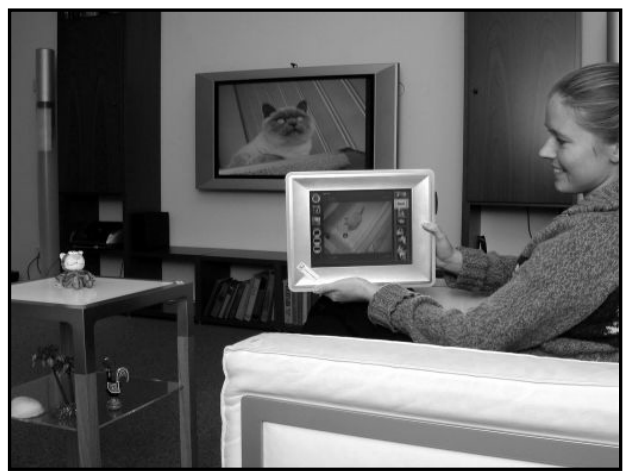

Fig. 2. The Digital Photo Browser, the Chameleon Table and some graspable objects.

\section{Graspable User Interface Categories}

Ullmer and Ishii [41] published their "emerging frameworks for tangible user interfaces", giving a complete overview of different aspects of TUIs, amongst others describing an interaction model, application domains and an overview of four categories of TUI-instances. Those four categories can be divided into two groups, namely one group in which physical objects are used independent of each other and the second group consists of groups of physical objects which together create an object language. The independent object-group is called "associatives", representing physical objects which are individually associated with digital information (e.g., each sticker represents a URL such as in Ljungstrand et al., 2000), such as the souvenirs described in Section 2. The second group contains physical objects that rely upon other physical objects to create added value or meaning, such as spatial interpretations (e.g., a 2D layout such as in BuildIt [12]), constructive (e.g., for building 3D physical models, such as LEGO-like Blocks [4] or relational ones (e.g., creating temporary relations between different physical objects, such as annotating videos with blocks [7].

The physical objects which form the graspable part of the user-interface are termed "iconic", by Ullmer and Ishii [41], when they share representational characteristics with their digital associations, or "symbolic" when those physical objects do not physically represent some property of the digital information. 
Other relevant definitions related to Graspable/Tangible UI come from Holmquist, Redström and Ljungstrand [17]. They came up with the names "containers", "tools", "tokens" and "faucets" for four types of physical objects. Containers are generic physical objects that can be associated with any type of digital information and the physical shape gives no clues on the associations made with the digital information, besides they are primarily used to move information between devices or platforms. Tools also do not show a relationship between the physical information and the digital information; they are physical objects that can manipulate digital information, which means they often represent computational functions. Tokens, on the other hand, do reflect with their physical appearance the digital information associated with it and they are used for accessing stored information. Faucets are devices that can present the digital information associated with tokens. Apparently, the authors see "reading the information from the tokens" and "presenting the information from the tokens" as something done in the same device, whereas the work in Section 2 shows it can be two different devices (respectively a table and a display), as well. Holmquist, Redström and Ljungstrand [17] also used the term "overloading", by which they mean that one token might be associated with more than one piece of information. This overload of information might require the token to be location or context sensitive, showing particular pieces at particular locations or particular contexts, or a user might be able to access several pieces of information at the same time by means of a faucet.

According to Ullmer and Ishii [41] their term iconic is similar to Holmquist et al.'s [17] token and symbolic is similar to container. Only the meaning of the terms iconic and symbolic are limited to the physical representation of the associated digital information, whereas the terms token and container also are defined as giving information on the function of the objects as well (respectively: accessing stored information and moving information between devices and platforms).

Dourish [9] describes a categorization of "meaning-carrying" of Tangible User Interfaces. He starts by subdividing the objects into iconic and symbolic and the meaning of the objects is identified as either related to other physical objects or to actions. This can get complicated. Imagine, for example, the souvenirs of section 2.2. These souvenirs are objects, which link to memories mentally (perhaps those memories contain both objects and actions), but virtually those souvenirs link to digital photos that can be displayed by performing an action with the object. Dourish calls this "a blend of properties" (p. 168), which has potential but should be worked out in more detail.

A recent paper by Ullmer et al. [44] mentions token+constraint interfaces, where the constraints are regions that map tokens to digital information. An example of such a token+constraint system is the Chameleon Table together with souvenirs (see Section 2.3), although it is different from Ullmer et al.'s description since a token is used to associate and manipulate the constraints. In this example a token associates, the constraints are manipulated by another physical object (the Digital Photo Browser) and the table sets the constraints by its color instead of movement or physical location of the tokens. 


\section{Graspable User Interface Category Extension}

Although the division into "object-language" categories is extremely useful [41], it misses out some of the other dimensions of Graspable UIs that are particularly important for the associative-TUIs group. And since Ullmer and Ishii left out this category in a later publication [42] because they were "less confident of the utility of this category" [41], an extension to the categorization is proposed in this paper which both involves the spatial, constructive and relational TUIs as well as the associative TUIs invented by [41].

The extension of the TUI-instances or Graspable UI categories is based on the idea that users of personal Graspable UI objects have an existing mental model of the links between their personal physical objects and the associated digital information. A mental model in this paper stands for a link between objects and media that is not determined by the object's physical properties, but by past events known to the user in which these objects played a role, such as buying a souvenir in a far-away country or leaving usage traces on a piece of furniture during that party you also have photos of. Later those physical properties might remind the user of the links. This definition, for example, would exclude a physical object that looks like a book which is assumed by a user to have stories attached to it. After some experience with this book the user does have a mental model of the object and its associated media, but these relationships were not present from the beginning, they were learned.

Examples of studies in this area include the souvenirs mentioned in Section 2, but also Rosebud [13] [14] POEMs [39] and Passage [37]. The use of these Graspable UIs is more suitable for novice instead of expert users, according to the negligible learning time needed to create an internal association with an external object or a mental model. Therefore, the category extension starts with a subdivision of physical objects in "physical objects which have personal meaning to the user" (where the user is probably also the owner) and "physical objects that do not have personal meaning to the user" (where the user is "only" a user). This distinction immediately shows that the first group in particular seems very suitable for the home environment, since this is the place where most people keep their personal belongings. The second group is more suitable for expert users, since they are more willing to learn the relationships between physical objects and their digital associations. Therefore this group seems more useful in the office environment. Physical objects in the first group are mostly used by one person whereas objects in the second group can be used by a group of people.

The second subdivision is made based on the concept of "dynamic binding" [41], which means that digital associations can be created and thus changed or deleted by the user. One group of Tangible UIs does not support dynamic binding; they are termed "fixed" associations, while the other group can have "flexible" associations. It turns out that the examples of the Tangible UIs with fixed associations always have only one association, but that the flexible group often supports overloading (see Table 1 for examples). Both of these groups can be subdivided into "random" or symbolic associations, which for "random" associations means that the physical properties of the object do not represent the digital properties in any way, and for "meaningful" or iconic associations that there is a relationship between the physical and the digital information. 
All physical objects with a fixed association appear to fall under the tool category as defined by Holmquist et al. [17]. Therefore, this group can be subdivided into symbolic tools (e.g., music coming out of a bottle when open in musicBottles) and iconic tools (e.g., a glass lens-shaped object, which functions as a lens for beams of light in Illuminating Light [45]).

Fitzmaurice [10] came up with defining properties of Graspable User Interfaces, of which the following property seems to be most relevant for this paper:

Both input and output of such an interface should be space-multiplexed instead of time-multiplexed. Space-multiplexed indicates that every function (onscreen) has a physical device associated with it. On the other hand, the timemultiplexed PC-mouse is constantly reassigned to a new function (strongspecific versus weak-general devices).

According to Fitzmaurice [10] physical objects that support more than one function are time-multiplexed, which makes them fall outside the definition of a Graspable UI, which should be space-multiplexed. Perhaps it is interesting to note that spacemultiplexed means that each physical object can only have one function, but it can contain more media files at the same time. Take, for example, the Rosebud system [13] [14], where each stuffed toy can contain one or more stories told by the owner, but it still has this one function: retelling stories.

Although Holmquist et al.'s [17] token was meant to be "iconic", based on its function, which is accessing stored information, a new subdivision can be made, namely symbolic versus iconic tokens, because examples of both types of tokens exist. E.g., in the metaDESK system blocks are designed to look like miniature buildings because they represent them [40], and because these objects show their link between physical appearance and digital information they are iconic tokens. Symbolic tokens only reflect to its current users the associated digital information, e.g., attaching a TV-show to a pen [35]. Holmquist et al. [17] used the term container for both "symbolic associations" and for supporting overloading, which does not fit in the distinction made in this paper that flexible associations can support one or more associations and thus do not always support overloading, therefore the term container is not taken forward in this categorization.

In Table 1 all this information can be found, together with some examples of Graspable/Tangible UIs. In this table the different categories by Ullmer and Ishii [41] can also be placed, namely: constructive and relational TUIs belong in the box generic symbolic tools. The spatial TUIs belong in the box generic iconic tools and generic symbolic tokens, and the associative TUIs belong in the four boxes with flexible digital associations.

One Graspable UI can contain physical objects from more than one category, although the majority only consists of one category, exceptions appear in the spatial and relational categories by Ullmer and Ishii [41], e.g., SiteView [5], which uses generic symbolic tools (e.g., the "rain interactors" is a generic object showing text) and generic iconic tools (e.g., the "lights on interactor" is an object shaped as a light). The Senseboard by Jacob et al. [25] contains both generic symbolic tools and generic symbolic tokens. The tokens are fridge magnet-like blocks that can be linked to one conference paper on the Senseboard; the tools can be used to execute commands on the tokens, such as "copy" or "link". And a third example, BuildIt [12] contains both generic iconic tools (a camera-shaped brick is used for determining a camera view 
onscreen) as well as generic symbolic tokens (rectangular bricks represent handles to pieces of furniture).

Table 1. An extension to the TUI-categorization by Ullmer and Ishii [41], with two dimensions: the type of physical objects and the type of digital associations with the physical object. The numbers between brackets are the number of associations possible with each physical object at the same time and $n=|n|$.

\begin{tabular}{|c|c|c|c|c|}
\hline \multirow{2}{*}{$\begin{array}{l}\text { Digital associations } \\
\text { Physical object type }\end{array}$} & \multicolumn{2}{|c|}{ Fixed (1) } & \multicolumn{2}{|c|}{ Flexible (n) } \\
\hline & $\begin{array}{c}\text { Symbolic } \\
\text { (tool) }\end{array}$ & $\begin{array}{l}\text { Iconic } \\
\text { (tool) }\end{array}$ & $\begin{array}{c}\text { Symbolic } \\
\text { (token) }\end{array}$ & $\begin{array}{l}\text { Iconic } \\
\text { (token) }\end{array}$ \\
\hline $\begin{array}{l}\text { No existing } \\
\text { mental model, } \\
\text { mostly multiple users = } \\
\text { Generic object }\end{array}$ & $\begin{array}{l}\text { Bricks (1) } \\
\text { DataTiles (1) } \\
\text { FRIDGE (1) } \\
\text { Logjam (1) } \\
\text { MetaDESK (1) } \\
\text { musicBottles (1) } \\
\text { MusiCocktail (1) } \\
\text { Navigational } \\
\text { Blocks (1) } \\
\text { PingPongPlus (1) } \\
\text { Senseboard (1) } \\
\text { SiteView (1) } \\
\text { Soundgarten (1) } \\
\text { Task Blocks (1) } \\
\text { Triangles (1) } \\
\text { Urp (1) }\end{array}$ & $\begin{array}{l}\text { ActiveCube (1) } \\
\text { BuildIt (1) } \\
\text { Illuminating } \\
\text { Light (1) } \\
\text { Lego-like } \\
\text { Blocks (1) } \\
\text { metaDESK (1) } \\
\text { Robotic toys (1) } \\
\text { SenseTable (1) } \\
\text { SiteView (1) } \\
\text { Urp (1) }\end{array}$ & $\begin{array}{l}\text { BuildIt (0/1) } \\
\text { InfoStick (n) } \\
\text { MediaBlocks (n) } \\
\text { "memory } \\
\text { objects" (n) } \\
\text { MusiCocktail } \\
\text { (n) } \\
\text { Rosebud (n) } \\
\text { Senseboard } \\
(0 / 1) \\
\text { TellTale (0/1) } \\
\text { Triangles (0/1) } \\
\text { WebStickers (n) } \\
\text { WWICE (0/1) }\end{array}$ & \\
\hline $\begin{array}{l}\text { With existing } \\
\text { mental model, } \\
\text { mostly single } \\
\text { user = } \\
\text { Personal object }\end{array}$ & & & Passage $(0 / 1)$ & $\begin{array}{l}\text { POEMs (n) } \\
\text { Phenom (n) } \\
\text { Living } \\
\text { Memory Box } \\
\text { (n) }\end{array}$ \\
\hline
\end{tabular}

\subsection{Generic Graspable User Interfaces}

Generic Graspable User-Interface objects are mostly designed for office environments. For example, blocks can be used as tools to control specific PC-functions, such as Lego-like Blocks [4], Navigational Blocks [6], Bricks [11], BuildIt [12], Senseboard [25], ActiveCube [26], SenseTable [31], DataTiles [34], Task Blocks [38], metaDESK [40], Urp [46] and FRIDGE [47]. Besides for tools generic Graspable-UI blocks can also be used as tokens that contain information or files, such as infoStick [27], MediaBlocks [43], WWICE-tokens [35] and WebStickers [28]. Examples outside offices include toys, such as TellTale [3], Triangles [15], PingPongPlus [24], robotic toys [30], Rosebud [13] [14] and "memory objects" [16]. Besides toys a number of papers focused on audio or video applications in the home, such as Logjam [7], musicBottles [22], MusiCocktail [29] and soundgarten [48]. 


\subsection{Personal Graspable User Interfaces}

Most personal Graspable UI objects, which are all tokens, can be found in home environments, with the exception of the personal symbolic tokens of Passage [37]. Since Graspable User Interfaces in the home can be used by any type of user, e.g., people who do not have any PC-experience, the system and in particular the graspable objects should make clear, in one way or another, how they should be used. It is most clear to the user if she can come up with the digital associations and the graspable objects, such as in POEMs [39], the Living Memory Box [36] and the souvenirs of Section 2.

Although Ullmer and Ishii [41] do not address "overloading" in their "emerging frameworks"-paper, this concept might explain partly the usefulness of their so-called "associative" category (besides the statement already made about the use in-home). Ullmer and Ishii state that they "are less confident of the utility of this category than those we have considered thus far. Nonetheless, the instances we have identified do seem to exhibit some consistency, suggesting the category may have merit". (The categories considered thus far stand for the spatial, constructive and relational systems.)

To the authors' knowledge all personal iconic tokens of the "associative" systems support "overloading", including the Graspable UI described in Section 2 of this paper, which incorporates souvenirs that link to related media-items. This might explain why the iconic tokens of "associative systems" are not used for complex tasks involving other tokens: the digital interface is already complex enough with multiple pieces of information associated with each token.

\section{Discussion}

The strength of the extension proposed in this paper is that it includes Graspable UIs, which make use of existing everyday graspable object like the personal souvenirs people have in their homes. The need for this category of Graspable User Interfaces is supported by recent views on the future of computing, such as Ambient Intelligence [1][2]. These visions state that in the future many networked devices will be integrated in the environment and the numerous examples of personal tools and tokens that are featured in these future scenarios show that this can be done with personal objects people already have.

Therefore, an interesting area of future research would be the personal objectgroup. Currently many case studies start with personal objects and later "upgrade" them with a link to digital information. Is it possible to do this the other way around, or will this inhibit the personalization of the object? And why are the personal-fixed toolboxes empty, because the field is not yet mature, does not exist long enough? One can imagine a personal tool such as a bowl, which represents the user. Each object, e.g. a souvenir like the ones described in Section 2, that is placed in this bowl links to the digital associations created by the "bowl owner", since one souvenir can have different associations for different users. If the bowl represents its filtering function in 
one way or another, e.g., by text, icons or perhaps shape, it would be a personal iconic tool, and if it does not it would be a personal symbolic tool.

An interesting finding is that all the Graspable UIs mentioned in the personal iconic token box (Table 1) appear to make use of external memory (a subset of distributed cognition, see e.g. [32]), although none of the papers mentions this explicitly. This is not possible with generic objects, since they are all alike, but it is convenient for personal objects, because the mental model is created by the user himself and not imposed by the system. Therefore this group of objects seems very suitable as reminders. Another interesting finding is that currently no Graspable UIs are known in the personal fixed categories.

The Generic Iconic Token box in Table 1 shows no examples, perhaps because an object that is flexible in its associations can contain several links but also several types of media and it is hard to represent an ever changing media type.

Another remark concerns Dourish [9] who talked about dividing Tangible UIs according to the meaning they carried, on a scale from objects to actions. This scale might be most useful for the generic objects presented in this paper, since they have a unified meaning to users. The personal objects with predefined mental models might be difficult to fit in this subdivision.

\section{Conclusions}

This paper explains a possible extension to the TUI-categorization by Ullmer and Ishii [41]. The extension is based on the idea that users of personal objects have an existing mental model of the links between their personal physical object and the accompanying digital information. To the authors' opinions this extension is valuable, since the associative TUIs fit in, which Ullmer and Ishii [41] found hard to categorize. Furthermore, the benefit of using personal objects instead of generic objects is that in the first instance users already have mental models, and the Graspable or Tangible User Interface can support existing object systems, instead of designing new ones that have to be learned by users.

Acknowledgements. The authors would like to thank the other members of the project team that created the Digital Photo Browser and the Chameleon Table: E. Dijk, N. de Jong, E. van Loenen, D. Tedd, D. Teixeira and Y. Qian.

\section{References}

1. Aarts, E., Harwig, R., and Schuurmans, M. (2001). Ambient Intelligence. In P.J. Denning (Ed.) The Invisible Future, New York, USA: ACM Press, 235-250.

2. Aarts, E. and Marzano, S. (2003). The New Everyday, Views on Ambient Intelligence, Rotterdam, The Netherlands: 010 Publishers. 
3. Ananny, M. (2002). Supporting Children's Collaborative Authoring: Practicing Written While Composing Oral Texts, Proceedings of Computer-Supported Collaborative Learning Conference (CSCL2002), 585-596.

4. Anderson, D, Frankel, J., Marks, J., Leigh, D., Sullivan, E., Yedidia, J. S. and Ryall, K. (1999). Building Virtual Structures with Physical Blocks, Proceedings of User Interface Software and Technology (UIST'99), 71-72.

5. Beckman, C. and Dey, A. K. (2003). SiteView: Tangibly Programming Active Environments with Predictive Visualization, Adjunct Proceedings of UbiComp 2003, 167-168.

6. Camarata, K., Yi-Luen Do, E., Johnson, B. R. and Gross, M. D. (2002). Navigational Blocks: Navigating Information Space with Tangible Media, Proceedings of the Intelligent User Interface Conference (IUI'02), 31-38.

7. Cohen, J., Withgott, M. and Piernot, P. (1999). Logjam: A Tangible Multi-Person Interface for Video Logging, Proceedings of the ACM Conference on Human Factors in Computing Systems (CHI'99), 128-135.

8. Dijk, E. O., Hoven, E. A. W. H. van den, Loenen, E. J. van, Qian, Y., Tedd, D., and Teixeira, D. (2000). A Portable Ambient Intelligent Photo Browser, Philips Internal Document, Koninklijke Philips Electronics N.V.

9. Dourish, P. (2001). Where the Action Is: The Foundations of Embodied Interaction, Cambridge, Massachusetts: MIT Press.

10. Fitzmaurice, G. W. (1996). Graspable User Interfaces, Ph.D. Thesis, Dept. of Computer Science, University of Toronto.

11. Fitzmaurice, G. W., Ishii, H. and Buxton, W. (1995). Bricks: Laying the foundations for Graspable User Interfaces, Proceedings of the ACM Conference on Human Factors in Computing Systems (CHI'95), New York, USA: ACM Press, 442-449.

12. Fjeld, M., Voorhorst, F., Bichsel, M., Lauche, K., Rauterberg, M. and Krueger, H. (1999). Exploring Brick-Based Navigation and Composition in an Augmented Reality, Proceedings of Handheld and Ubiquitous Computing (HUC'99), Lecture Notes in Computer Science, Berlin, Germany: Springer-Verlag, vol. 1707, 102-116.

13. Glos, J. and Cassell, J. (1997a). Rosebud: Technological Toys for Storytelling, Proceedings of the ACM Conference on Human Factors in Computing Systems (CHI'97), New York, USA: ACM Press, 359-360.

14. Glos, J. W., and Cassell, J. (1997b). Rosebud: A Place for Interaction Between Memory, Story, and Self, Proceedings of the 2nd International Conference on Cognitive Technology (CT'97), 88.

15. Gorbet, M. G., Orth, M. and Ishii, H. (1998). Triangles: Tangible Interface for Manipulation and Exploration of Digital Information Topography, Proceedings of the ACM Conference on Human Factors in Computing Systems (CHI'98), 49-56.

16. Holmquist, L. E., Helander, M. and Dixon, S. (2000). Every Object Tells a Story: Physical Interfaces for Digital Storytelling, Proceedings of NordiCHI2000, Retrieved 13 Oct 2003, from the website of Viktoria Institute's Future Applications Lab: http://www.viktoria.se/fal/ publications/play/2000/objectstory.pdf.

17. Holmquist, L. E., Redström, J. and Ljungstrand, P. (1999). Token-Based Access to Digital Information, Proceedings of the first international symposium on Handheld and Ubiquitous Computing, HUC'99, Springer-Verlag, 234-245.

18. Hoven, E. A. W. H. van den (2000). Recollecting Memories, Masters Thesis, Eindhoven University of Technology, The Netherlands, ISBN 90-444-0042-8.

19. Hoven, E. A. W. H. van den (2004). Graspable Cues for Everyday Recollecting, PhD Thesis, Department of Industrial Design, Eindhoven University of Technology, The Netherlands, ISBN 90-386-1958-8.

20. Hoven, E. A. W. H. van den, and Eggen, J. H. (2001). A Graspable User-Interface for an Ambient-Intelligent Photo Browser, Presented at the I3 Spring Days 2001, Porto, Portugal. 
21. Hoven, E. van den and Eggen, B. (2003). Digital Photo Browsing with Souvenirs, Proceedings of the Interact2003, 1000-1004. The accompanying video is available on CD together with the book-format proceedings and can be retrieved from: http://homelab.htc.nl.philips.com/movies/index.html (Philips Internal only).

22. Ishii, H., Mazalek, A. and Lee, J. (2001). Bottles as a minimal interface to access digital information, Proceedings of the ACM Conference on Human Factors in Computing Systems (CHI'O1), 187-188.

23. Ishii, H. and Ullmer, B. (1997). Tangible Bits: Towards Seamless Interfaces between People, Bits and Atoms. Proceedings of the ACM Conference on Human Factors in Computing Systems (CHI'97), 234-241.

24. Ishii, H., Wisneski, C., Orbanes, J., Chun, B. and Paradiso, J. (1999). PingPongPlus: Design of an Athletic-Tangible Interface for Computer-Supported Cooperative Play, Proceedings of the ACM Conference on Human Factors in Computing Systems (CHI'99), 394-401.

25. Jacob, R. J. K., Ishii, H., Pangaro, G. and Patten, J. (2002). A Tangible Interface for Organizing Information Using a Grid, Proceedings of the ACM Conference on Human Factors in Computing Systems (CHI'02), 339-346.

26. Kitamura, Y., Itoh, Y. and Kishino, F. (2001). Real-time 3D Interaction with ActiveCube, Proceedings of the ACM Conference on Human Factors in Computing Systems (CHI'01), 355-356.

27. Kohtake, N., Rekimoto, J. and Anzai, Y. (1999). InfoStick: an Interaction Device for Inter-Appliance Computing, Workshop on Handheld and Ubiquitous Computing (HUC'99), Retrieved 13 Oct 2003, from Sony's website: http://www.csl.sony.co.jp/person/rekimoto/papers/ InfoStick.pdf.

28. Ljungstrand, P., Redström, J. and Holmquist, L. E. (2000). WebStickers: Using Physical Tokens to Access, Manage and Share Bookmarks to the Web, Proceedings of Designing Augmented Reality Environments, DARE'00.

29. Mazalek, A. and Jehan, T. (2000). Interacting with Music in a Social Setting, Proceedings of the ACM Conference on Human Factors in Computing Systems (CHI'O0), 255-256.

30. Patten, J., Griffith, L. and Ishii, H. (2000). A Tangible Interface for Controlling Robotic Toys, Proceedings of the ACM Conference on Human Factors in Computing Systems (CHI'O0), 277-278.

31. Patten, J., Ishii, H., Hines, J. and Pangaro, G. (2001). SenseTable: A Wireless Object Tracking Platform for Tangible User Interfaces, Proceedings of the ACM Conference on Human Factors in Computing Systems (CHI'01), 253-260.

32. Perry, M. (2003). Distributed Cognition. In J. M. Carroll (Ed.), HCI models, theories and frameworks: toward a multidisciplinary science, San Francisco, USA: Elsevier Science, 193-223.

33. Philips Identification (n.d.). HITAG, Retrieved 13 Oct 2003, from Philips' Semiconductors website: http://www.semiconductors.philips.com/markets/identification/products/ hitag.

34. Rekimoto, J., Ullmer, B. and Oba, H. (2001). DataTiles: A Modular Platform for Mixed Physical and Graphical Interactions, Proceedings of the ACM Conference on Human Factors in Computing Systems (CHI'01), 269-276.

35. Sluis, R. van de, Eggen, B., Jansen, J. and Kohar, H. (2001). User Interface for an InHome Environment, In M. Hirose (Ed.), Human-Computer Interaction Interact'01, IOS Press, 383-390.

36. Stevens, M. M., Abowd, G. D., Truong, K. N., and Vollmer, F. (2003). Getting into the Living Memory Box: Family Archives \& Holistic Design, Proceedings of $1 A D$ (First International Conference on Appliance Design), (in press). 
37. Streitz, N., Geissler, J., Holmer, T., Konomi, S., Müller-Tomfelde, C., Reischl, W., Rexroth, P., Seitz, P. and Steinmetz, R. (1999). i-LAND: An interactive Landscape for Creativity and Innovation, Proceedings of the ACM Conference of Human Factors in Computing Systems (CHI'99), 120-127.

38. Terry, M. (2001). Task Blocks: Tangible Interfaces for Creative Exploration, Proceedings of the ACM Conference on Human Factors in Computing Systems (CHI'01), 463-464.

39. Ullmer, B. (1997). Models and Mechanisms for Tangible User Interfaces, Masters thesis, MIT Media Laboratory, Cambridge, USA.

40. Ullmer, B. and Ishii, H. (1997). The metaDESK: Models and Prototypes for Tangible User Interfaces, Proceedings of Symposium on User Interface Software and Technology (UIST'97), 223-232.

41. Ullmer, B. and Ishii, H. (2000). Emerging frameworks for tangible user interfaces, $I B M$ Systems Journal, 39(3-4), 915-931.

42. Ullmer, B. and Ishii, H. (2001). Emerging Frameworks for Tangible User Interfaces. In J. M. Carroll (Ed.), Human-Computer Interaction in the New Millenium, Addison-Wesley, 579-601.

43. Ullmer, B., Ishii, H. and Glas, D. (1998). MediaBlocks: Physical Containers, Transports, and Controls for Online Media, Proceedings of SIGGRAPH'98, 379-386.

44. Ullmer, B., Ishii, H. and Jacob, R. J. K. (n.d.). Token+constraint systems for tangible interaction with digital information (submitted).

45. Underkoffler, J. and Ishii, H. (1998). Illuminating Light: An Optical Design Tool with a Luminous-Tangible Interface, Proceedings of the ACM Conference of Human Factors in Computing Systems (CHI'01), 542-549.

46. Underkoffler, J. and Ishii, H. (1999). Urp: A Luminous-Tangible Workbench for Urban Planning and Design, Proceedings of the ACM Conference on Human Factors in Computing Systems (CHI'99), 386-393.

47. Vroubel, M., Markopoulos, P. and Bekker, M. (2001). FRIDGE: exploring intuitive interaction styles for home information appliances, Proceedings of the ACM Conference of Human Factors in Computing Systems (CHI'01), 207-208.

48. Wolf, M. (2002). Soundgarten: A Tangible Interface that Enables Children to Record, Modify and Arrange Sound Samples in a Playful Way, Masters thesis, University of Applied Sciences Cologne, Germany. 